\title{
Imaging with STEM Detector, Experiments vs. Simulation
}

\author{
F. Mika, C.G.H. Walker, I. Konvalina and I. Müllerová
}

Institute of Scientific Instruments of the ASCR, v.v.i., Královopolská 147, 61264 Brno, Czech

Republic.

Although there are a great many Scanning Electron Microscopes (SEMs) and Scanning Transmission Electron Microscopes (STEMs) currently in existence, obtaining quantitative information from the electron signal is proving difficult to establish [1]. In order to create good quantitative procedures, a comparison between experimental and theoretical signals from well defined samples needs to be carried out. Since the theoretical understanding of electron transport at low energies remains relatively poor, studies which make use of the higher energy scattered primary electrons would be a good place to start such a comparison. Hence, one could compare the signal obtained from a backscattered electron detector in an SEM with what one might expect from a Monte Carlo (MC) simulation. Another approach would be to consider the transmitted electrons (TEs) through thin films of elemental materials in a STEM. It is the latter approach that we will concentrate upon in this report. This is because calibrating the STEM detector using the primary beam is possible using the transmitted beam when no sample is present. However this approach is not possible for backscattered measurements. The aim of this work is to find out the angular and energy distribution of the TEs that are very important for the understanding of image formation in STEM.

The experiment was performed on the ultra-high resolution SEM, Magellan 400 [2], equipped with a multi-annular semiconductor STEM photodiode (PD). The PD is divided into six concentric annuli and imaged in bright field (BF), four dark field (DF1-4) and high angle annular dark field (HAADF) modes (see Figure 1). Other parameters were: beam current $=50 \mathrm{pA}$, scan speed $=10 \mu \mathrm{s}$, magnification $=50 \mathrm{k}$.

During the experimental study light and heavy element foils of 100nm thickness were imaged. The foils were made from silicon and gold. The primary beam energies used were $15 \mathrm{keV}$ and $30 \mathrm{keV}$ and we collected the image from each of six annuli and measured the average grey level of a defined image area to determine the signal intensity. The grey level of an image can be adjusted using Brightness and Contrast settings on the microscope. These settings modify the gain and offset of the PD amplifiers and hence it is best to keep them constant during a series of experiments. However, since the signal collected by the HAADF segment considerably exceeded that of other PDs (and to a lesser extent this also applies to the DF4 PD), a procedure needed to be adopted so that the HAADF and DF4 signals could be compared with the signals from the other PDs.

Method of acquiring data and calibrating PDs: The sensitivity of each PD was calibrated by directing the primary beam on to each segment of the STEM PD in turn. Since the signal was so much stronger when the beam strikes the PD directly, a different contrast setting was used to acquire this signal. However, the contrast was the same (40\%) for each PD for the $100 \%$ signal calibration. The $0 \%$ signal was determined by acquiring signals from each PD when the beam was blanked. The contrast settings for the blanked beam were then same as used during acquisition of the signal through the films. This gave a $100 \%$ and $0 \%$ signal for each segment of the PD. Only one section of the HAADF segment could be reached when the beam struck the PD directly, so it was assumed that the other segments of the 
HAADF had the same amplifier gain and offset. The estimated output of each PD (OUT) could be estimated by using

$$
O U T=\frac{S-B}{D-B}
$$

where B is the signal when the beam is blanked, D is the $100 \%$ signal when the beam strikes the PD directly and $S$ is the signal acquired when the beam strikes the film. Only one segment of the HAADF was used when acquiring data from each sample. This enabled data from all the segments to be acquired for the same settings of Brightness and Contrast. The signals for the HAADF PD were multiplied by 6 in order to determine the HAADF signal from all HAADF sectors. It is possible that the use of different contrast settings for acquiring the $100 \%$ signal could also lead to some systematic error.

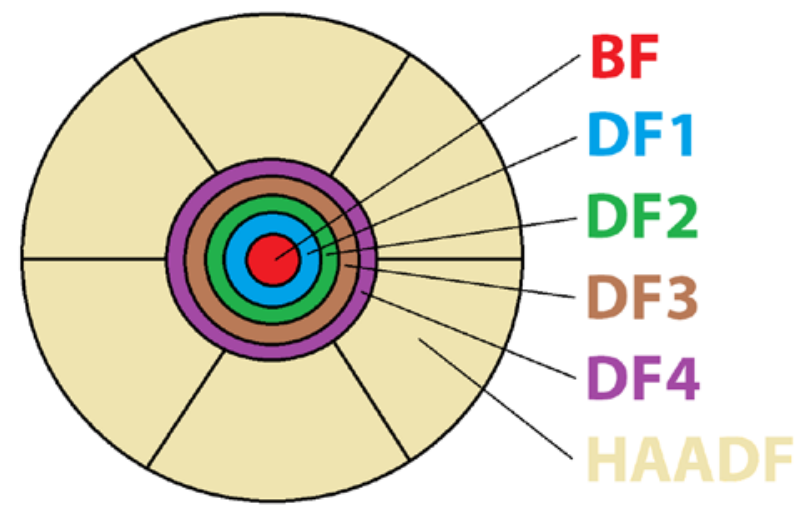

The outer radius of the HAADF is about $10 \mathrm{~mm}$ and the outer radius of DF4 is about $2.5 \mathrm{~mm}$ with the other PDs approximately equally spaced within DF4. The HAADF annulus is subdivided into six angular segments.

Figure 1. Schematic drawing of the STEM III photodiode.

Since the output from the PDs could not be determined from the image intensities, only the ratios of the signal from each dark field segment (i.e. DF1, DF2 etc) with the BF signal are considered. The errors in the experimental values are estimated from the standard deviation in the image intensities.

The electron trajectories were simulated using MC [3] and since the experiments were carried out with the immersion field switched on, the trajectories of the electrons after they exit from the underside of the foil sample were traced in the magnetic field in free space using the software package EOD [4]. The total energy passed to the each of the STEM PDs was determined by its radius from the optic axis. These signals were the stored and modified by a sensitivity function to account for the dead layer at the surface of the PD. The simulated signal acquired by each PD was then ratioed with the signal from the $\mathrm{BF}$ PD so as to compare with the measurements. Errors in the simulated results were estimated from the statistics of the MC results.

Figure 2 and Table 1 show the signal obtained from each PD (ratioed with the BF signal) for both experimental and simulated results. The experiments and simulations appear to show similar trends in both Si and Au samples. For example, in the case of Si 30kV, the signal initially drops from BF to DF1 and DF2 and then rises again for both simulation and experiment. However, the simulation shows a much stronger rise in signal towards the high scattering angles such as the HAADF as compared to the 
measurements. This difference could be due to the difficulty in establishing a reliable quantification procedure by the use of calibration with a known electron signal as outlined earlier.

Since there is disagreement especially for high angles further investigations into the energy of the electrons striking each PD was undertaken by determining the energy spectrum of the electrons striking each PD in the MC simulation. This can be expressed by the function $\mathrm{N}(\mathrm{E})$, where $\mathrm{N}$ is the number of electrons and $\mathrm{E}$ is the energy. However, the PDs measure the energy of the each electron striking the PD and not the electron current striking the PD.

Hence, each spectrum was multiplied by the energy, resulting in a spectrum EN(E). Each spectrum was then divided by the total number of electrons simulated, so they show the average spectrum per electron. Such spectra are shown in Figure 3. The number of bins in each spectrum is 100, so the energy width of each bin is $150 \mathrm{eV}$ for $15 \mathrm{keV}$ primary energy and $300 \mathrm{eV}$ for $30 \mathrm{keV}$ primary energy. Hence, if for example one considers the HAADF spectrum in Figure 3(a) then the maximum signal is about $400 \mathrm{eV}$ for electrons between $14.45 \mathrm{keV}$ to $14.6 \mathrm{keV}$. Therefore for each primary electron, on average $400 \mathrm{eV}$ will be deposited in the HAADF PD for electrons in the range $14.45 \mathrm{keV}$ to $14.6 \mathrm{keV}$. The integral under each curve gives the simulated signal for each PD. Figure 4 gives another representation for the landing positions in terms of the distance of the landing position on the y axis versus the energy of the electron on the $\mathrm{x}$ axis. The landing positions are strongly influenced by the magnetic field of the objective lens which prevents the electrons straying too far from the optic axis. One can also see from Figures 3 and 4 that for $\mathrm{Au} 30 \mathrm{keV}$, Si $30 \mathrm{keV}$ and Si $15 \mathrm{keV}$, the large majority of the signal comes from electrons with energy only slightly removed from that of the primary beam.

In all cases, the experiment results drop going from BF to DF1 and then rise for each PD until the HAADF PDs. This effect is repeated in the simulations except for $\mathrm{Si} 15 \mathrm{keV}$ which rises continuously from BF to HAADF. Hence, the trends of both experimental and simulation results are the same except for the Si $15 \mathrm{keV}$ DF1 point. However, typically the measured experimental data is significantly lower then the simulated data in the HAADF segment (by a factor 3 to 4) and to a lesser extent for the DF4 signal (by a factor 2 to 3.5). The cause for this discrepancy is currently unknown. The results shown in Figure 3 reveal that the majority of the electrons landing on the PDs have energies close to the primary beam energy except for $\mathrm{Au} 15 \mathrm{keV}$ where lower energy electrons contribute significantly to the BF and inner DF PDs. This is due to the confinement effect that is particularly strong for lower energy electrons (see Figure 5). By considering Figure 4(b), one can see that a large number of electrons are landing close to the outer edge of the HAADF PD $(10 \mathrm{~mm})$ for Au $30 \mathrm{keV}$ and only a small increase in the landing distance from the optic axis will reduce the HAADF signal for Au. The magnetic field has a confining effect on the electrons even at the highest energies (Figure 5). Hence, if the magnetic field were weaker, the electrons would be striking the PD at higher radii from the optic axis. This could result in a lower electron flux for the HAADF PD. However, those electrons striking the BF or inner DF PDs would also start to move with a higher radius of curvature in lower magnetic fields. 


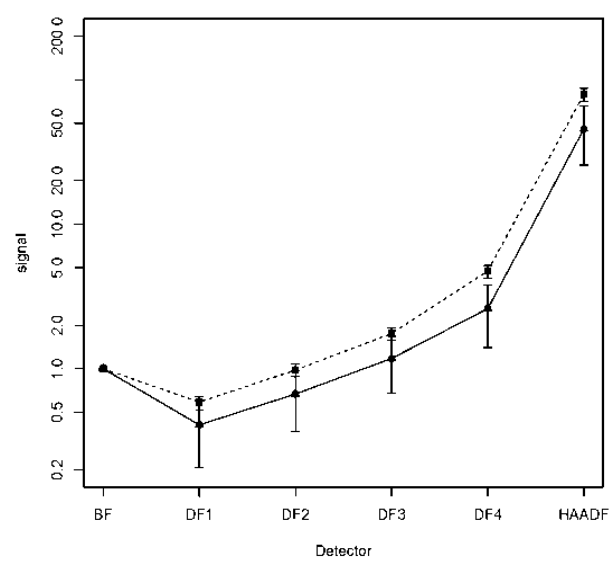

(a) Au $15 \mathrm{keV}$

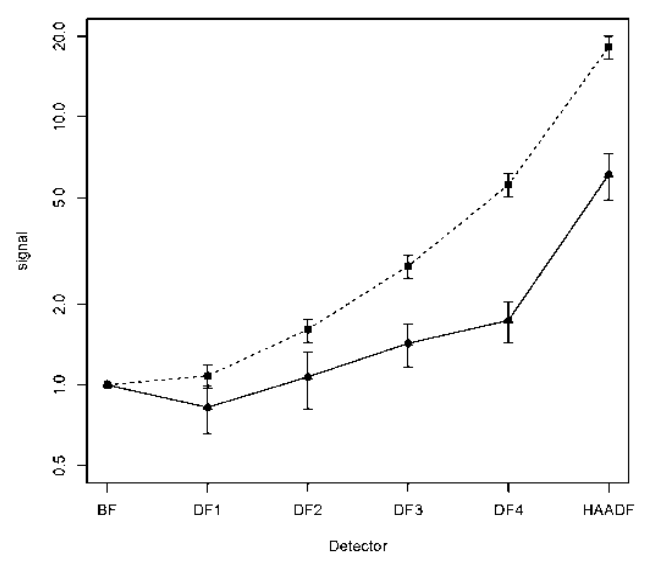

(c) Si $15 \mathrm{keV}$

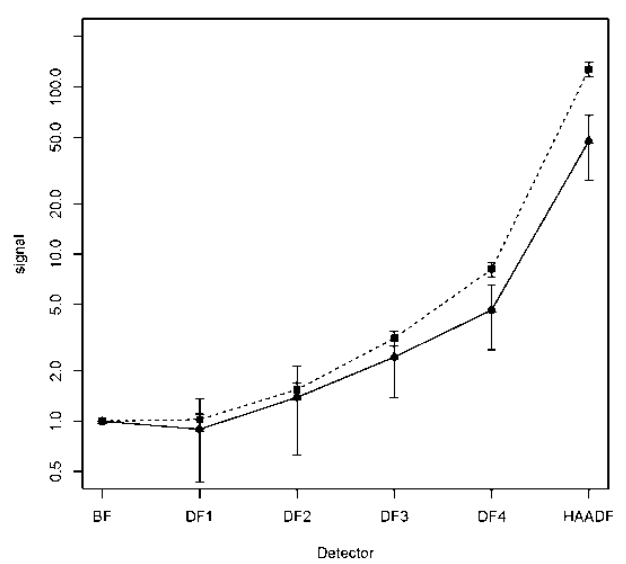

(b) Au $30 \mathrm{keV}$

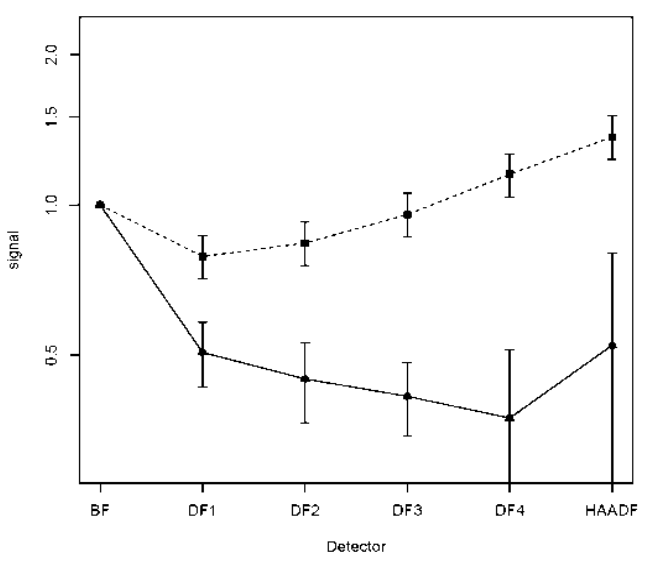

(d) Si $30 \mathrm{keV}$

Figure 2. Values for the ratio of signal from each of the PDs ratioed with the signal from the BF PD for experiment and the simulation. Note: Log vertical scale as signal values have a large range. Triangles and Solid line $=$ Experiment, Squares and Dotted line $=$ Simulation .

\begin{tabular}{|l|l|l|l|l|l|l|l|l|}
\hline & \multicolumn{3}{|l|}{ Si $15 \mathrm{keV}$} & \multicolumn{2}{l|}{ Si $30 \mathrm{keV}$} & \multicolumn{2}{l|}{ Au $15 \mathrm{keV}$} & \multicolumn{2}{l|}{ Au $30 \mathrm{keV}$} \\
\hline & E & S & E & S & E & S & E & S \\
\hline BF & 1.0 & 1.0 & 1.0 & 1.0 & 1.0 & 1.0 & 1.0 & 1.0 \\
\hline DF1 & 0.82 & 1.1 & 0.51 & 0.79 & 0.41 & 0.59 & 0.90 & 1.0 \\
\hline DF2 & 1.1 & 1.6 & 0.45 & 0.84 & 0.67 & 0.98 & 1.4 & 1.5 \\
\hline DF3 & 1.4 & 2.8 & 0.41 & 0.96 & 1.2 & 1.8 & 2.4 & 3.1 \\
\hline DF4 & 1.7 & 5.6 & 0.37 & 1.2 & 2.6 & 4.7 & 4.6 & 8.1 \\
\hline HAADF & 6.1 & 18.3 & 0.52 & 1.4 & 45 & 79 & 47 & 127 \\
\hline
\end{tabular}

Table 1. The data for Si and Au shown in Figure 2 shown in tabular form. In the second row the letters refer to the experiment $(\mathrm{E})$ or Simulation (S). 
If the magnetic field remained high near to the optic axis, but had reduced strength in regions away from the optic axis, the DF4 and HAADF signals would be reduced in comparison to the BF signal. This may explain the observed differences between the experimental and simulated results. Direct measurements of the magnetic field within the SEM would be the best way to determine if this is a valid explanation for the discrepancies. Other possible effects such as a carbon layer on the top and bottom of the films, or crystallinity of the films are thought to be insufficient to explain the large differences, but need to be considered. In addition, multiple scattering off the sample holder and other objects within the region between sample and PD will cause signal changes in all PDs (BF to HAADF).
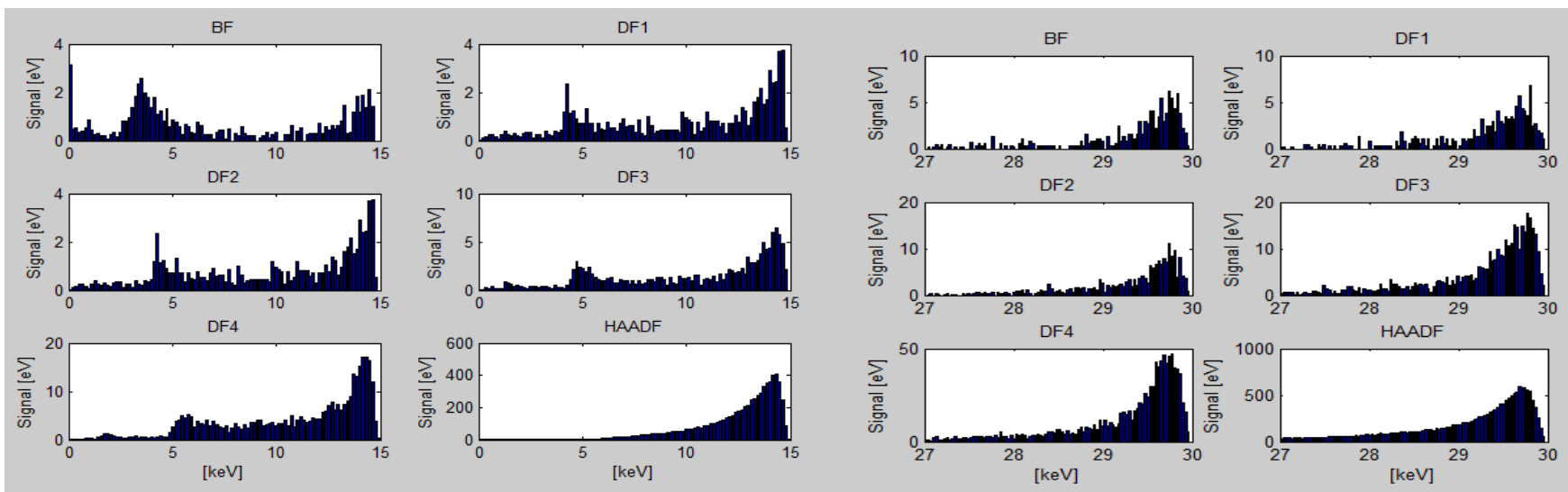

(a) Au at $15 \mathrm{keV}$ primary energy

(b) Au at $30 \mathrm{keV}$ primary energy


(c) Si at $15 \mathrm{keV}$ primary energy

(d) Si at $30 \mathrm{keV}$ primary energy

Figure 3. Energy histograms of the energy deposited per electron on the each segment of the STEM $P D$. It shows for silicon and gold sample how much signal in $\mathrm{eV}$ (Y axis) is deposited versus the energy of the detected TEs. Note that for figures (b), (c) and (d) the horizontal axis is expanded since there is little data at low energies. The number of bins in each case remains at 100, so the bin width for (c) and (d) is $10 \mathrm{eV}$ while that for (b) is $30 \mathrm{eV}$. However, careful characterisation of the magnetic field both on and off the optic axis within an SEM is vital for quantitative work. 


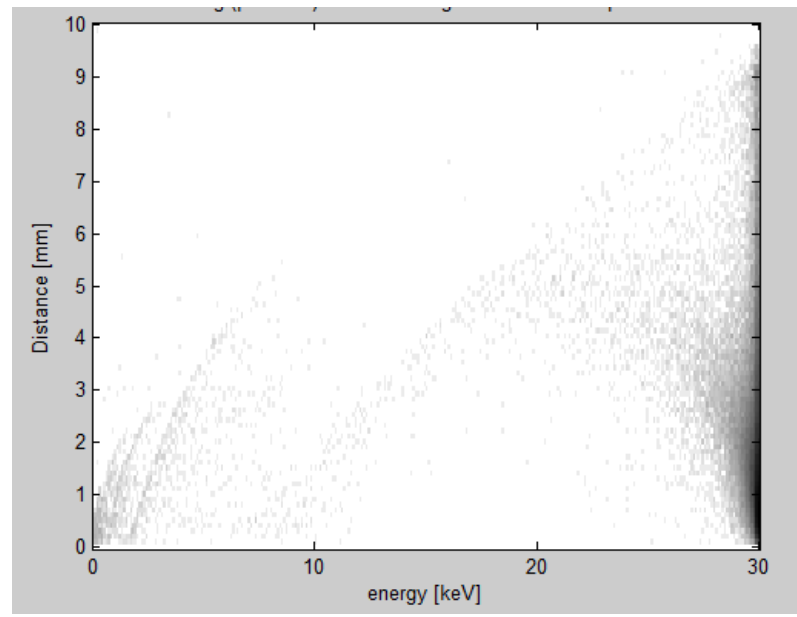

(a) $30 \mathrm{keV}$ primary beam, silicon

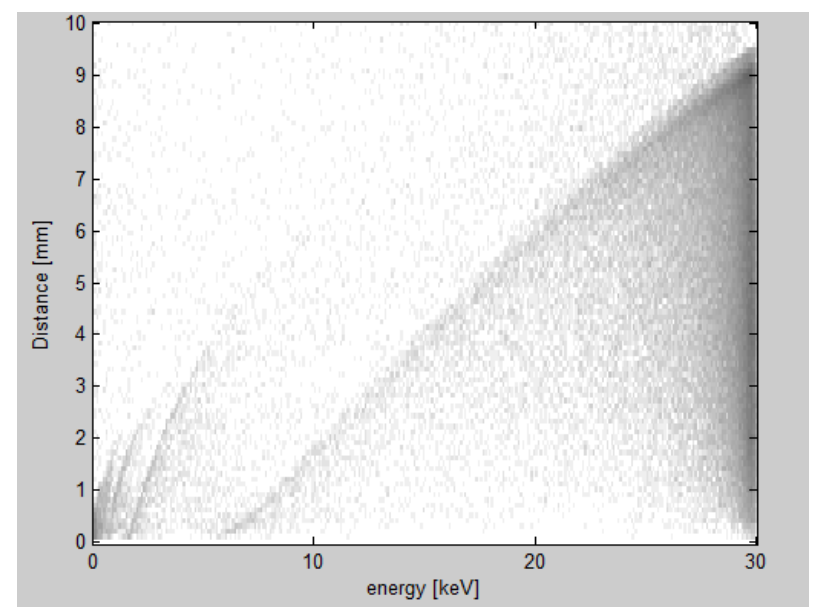

(b) $30 \mathrm{keV}$ primary beam, gold

Figure 4. Simulated results for the energy of the TEs impacted on the STEM PD plotted as a function of the radial position of impact on the STEM PD. Dark regions show high flux of impacting electrons.

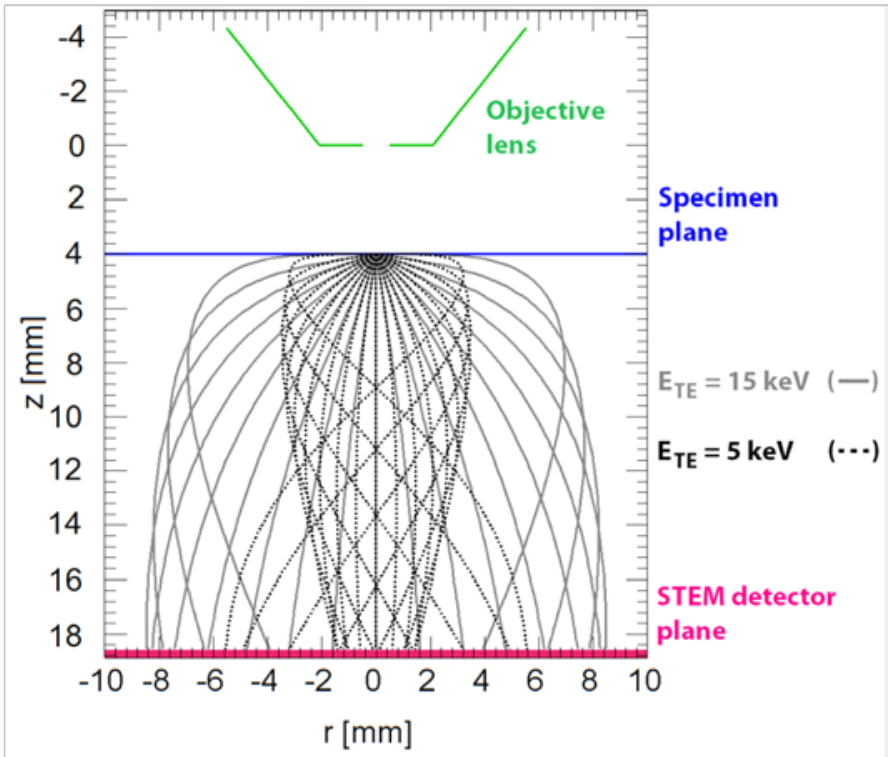

Figure 5. Trajectories of electrons between sample and PD for $15 \mathrm{keV}$ primary beam energy and TE energies of $15 \mathrm{keV}$ and $5 \mathrm{keV}$. When operating at $30 \mathrm{keV}$ primary beam energy, the $30 \mathrm{keV}$ TEs have exactly the same trajectory as the $15 \mathrm{keV}$ electrons shown here as the magnetic field is correspondingly magnified for higher primary beam energy.

\section{References:}

[1] SJ Pennycook, PD Nellist in „Scanning Electron Microscopy - Imaging and Analysis“, (Springer, Berlin).

[2] http://www.fei.com/

[3] E Kieft and E Bosch, J. Phys. D: Appl. Phys. 41 (2008), 215310.

[4] J Zlamal and B Lencova, Nucl. Instrum. Meth. A 645 (2011), p. 278.

[5] The work is supported by the TA CR (TE01020118), MEYS CR (LO1212), its infrastructure by MEYS CR and EC (CZ.1.05/2.1.00/01.0017) and by ASCR (RVO:68081731). The authors would like to thank L. Frank and V. Krzyžánek for useful discussions and to A. Paták for his assistance with the experiments. 